\title{
A Self-Calibration Method for Boresight Error of Mobile Mapping System
}

\author{
Yunzhi $\mathrm{Li}^{1}{ }^{1, *}$, Yuntian Bai ${ }^{1}$, and Mengke Wang ${ }^{1}$ \\ ${ }^{1}$ College of Geodesy and Geomatics, Shandong University of Science and Technology, Qingdao 266000, Shandong, China
}

\begin{abstract}
The boresight error is one of the main error sources of the Mobile Mapping System(MMS), which directly affects the overall accuracy and quality of the laser point cloud. Unideal boresight error estimation seriously reduces the point cloud registration accuracy. When there is a feature place in the laser point cloud, the boresight error will cause the feature plane to deviate from the real plane. In order to solve the above problems, a visual axis calibration method for a mobile surveying and mapping system based on known feature planes is proposed. According to the plane features of 3D scene, the Gauss-Helmert adjustment model is established, and the normal equation for calculating the boresight error is derived. This is a simple and reliable calibration method.
\end{abstract}

\section{Introduction}

Mobile Mapping System(MMS) as a kind of the cutting edge of 3D spatial information data acquisition method, it is equipped with various sensors such as integrated 3D Laser Scanning, Position and Orientation System (POS) and Panoramic Camera $^{[1]}$. Many technologies such as multi-sensor time and space synchronization and multisource data fusion technology are used. MMS can obtain the laser point cloud of the ground object and synchronously collect its pose information with the integrated navigation system, so as to realizing the measurement of ground objects' position. It has many advantages such as fast, accurate, comprehensive, low cost and high degree of automation in obtaining information ${ }^{[2]}$. It is widely used in many fields such as digital city, street view map, urban component survey, road detection and feature extraction, 3D modelling. It can be predicted that the continuous improvement of the precision of mobile measurement system is the inevitable trend of future development ${ }^{[3-5]}$.

The MMS is a multi-sensor integrated system, and each sensor has a different coordinate system, in the integration, the inherent installation limitation of the whole system, so that the laser scanner and POS can not be installed accurately according to the drawings ${ }^{[6]}$. The MMS must be accurately calibration before it is applied. The traditional boresight calibration is to use point cloud visualization software to continuously adjust external parameters through manual operation until the point cloud of the same name repeatedly scanned by the Mobile Mapping system in different directions visually coincides ${ }^{[7]}$.In addition, the most commonly used methods for boresight calibration error of Mobile Mapping system are as follows. The Iterative Closed Point (ICP) algorithm solves the boresight calibration error of the MMS through an iterative registration process for the repeatedly scanned point cloud of the same name. For example, the ICP algorithm is used between two sets of point clouds with overlapping ranges, a rigid body transformation matrix is obtained through an iterative registration process, and the transformation matrix is applied to the overlapping point cloud error analysis model to complete boresight calibration $^{[8]}$. However, the ICP algorithm requires multiple iterations, and the amount of laser point cloud data obtained by MMS is generally large, so this method has a very large amount of computation and has certain requirements on time and hardware. The other is to extract the coordinates of the feature points pre-arranged in the calibration field from the point cloud data measured by the MMS and compare them with the real coordinates measured by the Electronic Total Station, so as to solve the unknown boresight parameters by the least square method $^{[9]}$. This method needs to accurately measure a large number of feature points, and the extraction of feature points mainly depends on manual operation, and is also greatly affected by vehicle speed and point cloud density, so it is difficult to extract accurately, which has a certain impact on the error results of boresight correction. In addition, the adjustment model is established by using the same plane characteristics of the MMS scanned in multiple directions, and the boresight parameters are calculated according to the least square method ${ }^{[10]}$. This method can only solve the boresight parameters, and the lever-arm error still needs to be calibrated by industrial measurement and other methods. A method for estimating the boresight of MMS based on the known feature plane is proposed. In this paper, theory of the method is described in detail, and the implementation process of specific inspection and calibration work is given, which is of practical significance for studying the calibration method of boresight of MMS.

\footnotetext{
* Corresponding author: 907276051@qq.com
} 


\section{The MMS and position equation}

\section{1 positioning equation}

we use the following point geo-referencing model :

$$
X_{e}=X_{o e}+R_{l}^{e} R_{b}^{l}\left(\left(I+\Omega_{b^{*}}^{b}\right) R_{b}^{b^{*}} X_{s}+l+\Delta l\right)
$$

Among them, $\mathrm{R}_{1}^{\mathrm{e}}, \mathrm{R}_{\mathrm{b}}^{\mathrm{l}}$ respectively represent the rotation matrix of local Cartesian coordinates coordinate system to the WGS-84 coordinate system, the rotation matrix of the carrier coordinate system to the local horizontal coordinate system. $\mathrm{R}_{\mathrm{b}}^{\mathrm{b}^{*}}$ is the approximate value of the rotation matrix from the laser scanner coordinate system to the carrier coordinate system. $\Omega_{\mathrm{b}^{*}}^{\mathrm{b}}$ represents the boresight error compensation matrix. $\mathrm{X}_{\mathrm{e}}, \mathrm{X}_{\mathrm{s}}$ are the coordinates of the data collected by the laser scanner in the WGS-84 coordinate system, and in the laser scanner coordinate system. $\mathrm{X}_{\mathrm{oe}}$ is the space coordinates of the origin of the local navigation coordinate system in the ECEF coordinate system. $l$ is the coordinate of the laser scanner coordinate system in the carrier coordinate system. $\Delta \mathrm{l}$ is the offset parameter of the boresight.

\subsection{Function model}

If a feature in the space can be described by a certain mathematical model, the characteristic equations can be expressed as a ternary $n$-degree polynomial. In the actual scene, this topic selects the common plane model. When the MMS acquires a known plane feature foot point $\left(\begin{array}{lll}X_{S} & Y_{S} & Z_{s}\end{array}\right)$, all of conditions are satisfied.

$$
a X_{i}+b Y_{i}+c Z_{i}-d=0
$$

Where: $a, b, c$ are the normal vector of the plane, and $\mathrm{d}$ is the distance from the origin to the plane.

When the plane parameters are known, the GaussHelmert adjustment model can be used to solve the boresight parameters and calculate the correction of the observations. The general linearized form of Gauss-Helmert adjustment model is:

$$
f(1, x)=A x+B v+w=0
$$

Where: $f(l, x)$ represents the adjustment function model, A represents the design matrix in which the function model calculates the partial derivative of the unknown parameters, $\mathrm{B}$ represents the design matrix after the partial derivative if the function model to the observation, $\mathrm{v}$ represents the correction of the observed value of the function model, and $\mathrm{w}$ is the closed difference vector of the function model.

When observations and unknown parameters can not be separated by transforming the functional relationship between them, the Gauss-Helmert adjustment model can be used to solve the optimization problem of a conditional equation with parameters.

$$
f(\hat{l}, \hat{x})=[a, b, c] \boldsymbol{X}_{\boldsymbol{e}}-d=0
$$

Among them, $X_{\mathrm{e}}$ is the coordinates of the data collected by the laser scanner in the WGS- 84 coordinate syste-m, a, b, c are the normal vector of the plane.

The model can simultaneously estimate the observed values, boresight parameters and characteristic parameter$\mathrm{s}$ of the MMS. The nonlinear observation equation is linearized by Taylor series expansion, and the general linearized form of Gauss-Helmert adjustment model is obtained.

$$
f(\hat{l}, \hat{x})=\left(l+\hat{v}, x^{0}+\hat{x}\right)=A \hat{v}+B \hat{x}+w=0
$$

Among them, $\hat{v}$ represents the number of corrections for observations, $\hat{x}$ correct the adjustment value for the boresight parameter, A represents the design matrix of the partial derivative of the control plane equation to the boresight parameters, $\mathrm{B}$ represents the design matrix of the partial derivative of the control plane equation to the observation, $\mathrm{w}$ is the closed difference vector of the control plane equation.

A new function can be constructed according to the least square method and Lagrange multiplier method.

$$
\Phi=\hat{v}^{\mathrm{T}} \mathrm{P} \hat{\mathrm{v}}-2 \mathrm{~K}^{\mathrm{T}}(\mathrm{A} \hat{\mathrm{v}}+\mathrm{B} \hat{\mathrm{x}}+\mathrm{w})
$$

Where: $\mathrm{P}$ is the weight matrix, $\mathrm{K}$ is the Lagrange multi-plier vector.

Find the first order partial derivative of $\hat{v}$ and $\hat{x}$ respect-ively, and make them equal to zero,that is :

$$
\left.\begin{array}{c}
\frac{\partial \Phi}{\partial \hat{v}}=2 \hat{v}^{T} P-2 K^{T} A=0 \\
\frac{\partial \Phi}{\partial \hat{x}}-2 K^{T} B=0
\end{array}\right\}
$$

and then the basic equations are given.

$$
\left.\begin{array}{c}
A \hat{v}+B \hat{x}+w=0 \\
\hat{v}=P^{-1} A^{T} K \\
B^{T} K=0
\end{array}\right\}
$$

Replace the second formula of formula (8) into the first formula .

$$
\left.\begin{array}{c}
A P^{-1} A^{T} K+B \hat{x}+w=0 \\
B^{T} K=0
\end{array}\right\}
$$

The corrected values of observed values and boresight parameters are obtained by solving the equation.

$$
K=-N_{a a}^{-1}(B \hat{x}+w)
$$

After that, it can be substituted into the second formula.

$$
\begin{gathered}
\hat{x}=-N_{b b}^{T} B^{T} N_{a a}^{-1} w \\
\hat{v}=-P^{-1} A^{T} N_{a a}^{-1} N_{a a}^{-1}(B \hat{x}+w)
\end{gathered}
$$

Among them, $\mathrm{N}_{\mathrm{bb}}=\mathrm{B}^{\mathrm{T}} \mathrm{N}_{\mathrm{aa}}^{-1} \mathrm{~B}, \mathrm{~N}_{\mathrm{aa}}=\mathrm{AP}^{-1} \mathrm{~A}^{\mathrm{T}}$.

\section{Experimental environment and data processing}

\subsection{Experimental hardware equipment}

This topic selects the portable MMS based on motorcycle, and the carrier is a motorcycle. It is mainly composed of data acquisition system, synchronous control system and data storage system, and integrates many kinds of sensors such as laser scanner, integrated navigation system, industrial camera and so on. The system has the characteristics of adaptability to the collection site, simple operation and flexibility. The initial boresight parameters between the laser scanner and the positioning and attitude system of the MMS used in the experiment are shown in Tab.1, and the initial external parameters contain large errors. The initial point clouds in the following are solved using this set of boresight parameters.

Tab.1 Initial external parameters of the system

\begin{tabular}{cccccc}
\hline \multicolumn{7}{c}{ Tab.1 Initial external parameters of the system } \\
\hline$\Delta \varphi$ & $\Delta \omega$ & $\Delta \kappa$ & $\Delta \mathrm{l}_{\mathrm{x}}$ & $\Delta \mathrm{l}_{y}$ & $\Delta \mathrm{l}_{z}$ \\
\hline 0 & -0.6 & 0 & 0.0028 & -0.056 & 0.054 \\
\hline
\end{tabular}




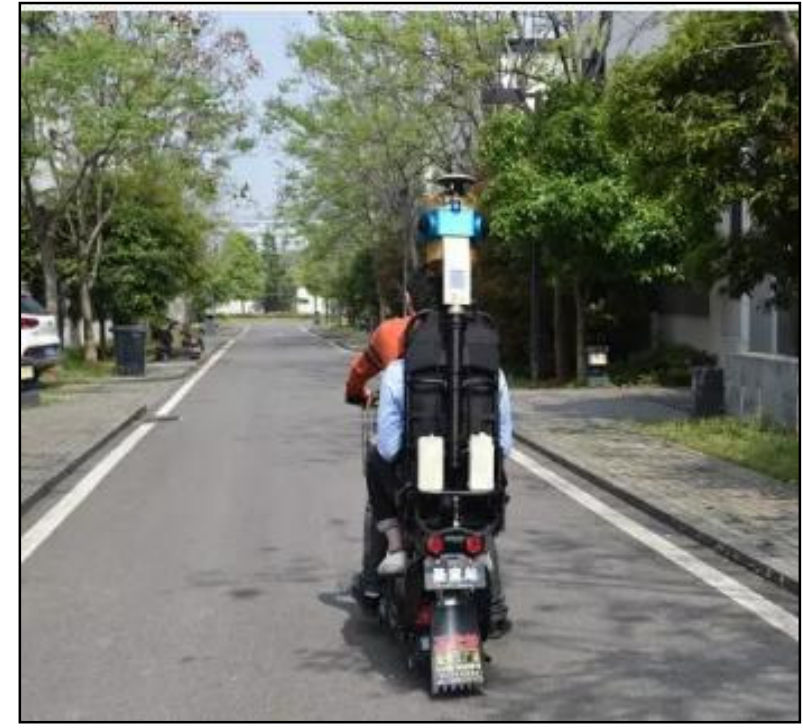

Fig.1. Portable Mobile Mapping system

\subsection{Feature plane extraction}

The traditional feature plane extraction method mainly depends on manual operation using visualization software. This method has the disadvantages of low efficiency and heavy workload. Before calibrating the boresight, the MMS obtains the initial boresight parameters according to the parameters of the design drawings or industrial measurement, but the accuracy of the initial point cloud is poor. In order to simplify the calibration process and improve the calibration accuracy, we can use the known plane parameters to complete the automatic extraction of the feature plane.

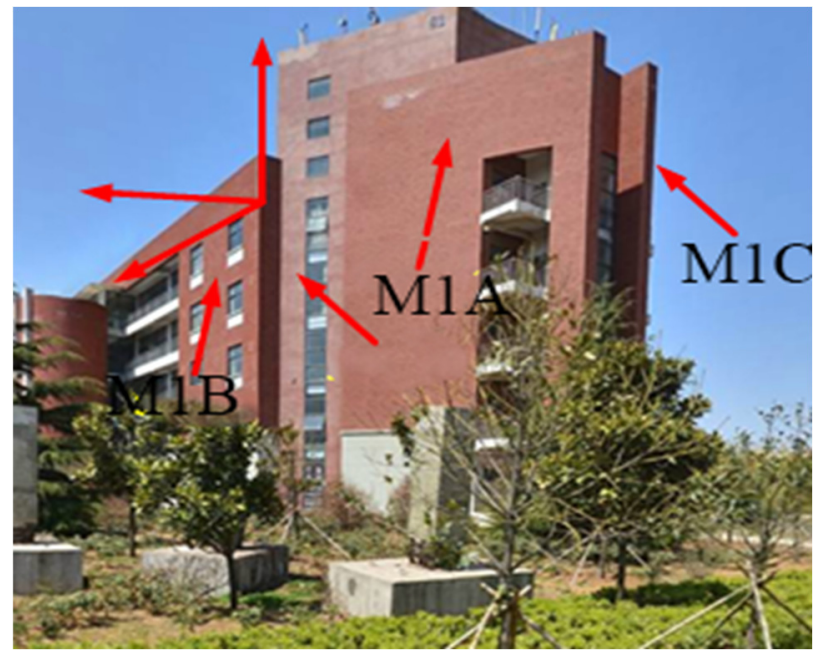

Fig.2. Plane selection

Based on the laser scanner and the feature plane, the point cloud date is substituted into the feature equation to obtain a point residual. If there are $n$ laser feet point $\left(x_{i} \quad y_{i} \quad z_{i}\right)$ and $t$ known planes $\left(a_{j}, b_{j}, c_{j}, d_{j}\right)$, by setting the threshold, the laser foot point whose residual error is less than the threshold is extracted. And when the density value is less than the threshold, it is classified as discrete point culling. the residual error is :

$$
w_{i}=\left(a_{j} x_{i}+b_{j} y_{i}+c_{j} z_{i}\right)-d_{j}
$$

Because the extraction of the feature plane is carried out strictly according to the plane equation, there are some discrete points which do not belong to the plane but are still coplanar with it, which are also regarded as local points, which need to be further eliminated. According to the parameters of the laser scanner equipped by the MMS, a reasonable radius density threshold is set, and the corresponding radius density is calculated for the local point of each feature plane extracted. When the density value is less than the threshold, it is classified as discrete point elimination. At this point, the extraction of the feature plane is completed.

\section{Calibration results and Analysis of boresight parameters of MMS}

The self-compiled calibration program is used to calculate the boresight parameters, and the iterative termination condition is that the correction number of the boresight angle is less than $1 \times 10^{-5}$, the error parameter of leverarm errors is less than $1 \times 10^{-4}$. The final calibration parameters after iteration are shown in Table. 2

Tab.2 Final calibration parameters

\begin{tabular}{cccccc}
\hline$\Delta \varphi$ & $\Delta \omega$ & $\Delta \kappa$ & $\Delta \mathrm{l}_{\mathrm{x}}$ & $\Delta \mathrm{l}_{y}$ & $\Delta \mathrm{l}_{z}$ \\
\hline 0.415 & -0.145 & 0.199 & 0.0037 & -0.018 & 0.096 \\
\hline
\end{tabular}

The laser point clouds of seven feature planes are corrected by the calibration parameters, and the residual errors of each feature plane and all feature planes before and after correction are calculated. The results show that the residual errors of each feature plane obtained by the MMS before inspection and calibration are large, but the accuracy of the system has been greatly improved after calibration. A shown in Tab.3.

Tab.3 Residual error before and after calibration

\begin{tabular}{cccc}
\hline ID & Points & $\begin{array}{c}\text { Before } \\
\text { calibration }\end{array}$ & $\begin{array}{c}\text { After } \\
\text { calibration }\end{array}$ \\
\hline M1A & 204653 & 0.181 & 0.015 \\
M1B & 415402 & 0.172 & 0.012 \\
M1C & 533331 & 0.168 & 0.018 \\
J1A & 342854 & 0.163 & 0.012 \\
J1B & 545612 & 0.172 & 0.015 \\
\hline
\end{tabular}

The residual values before and after laser foot calibration on each feature plane are counted respectively, and the histogram of residual distribution is drawn, as shown by Fig.3 to Fig10 . 


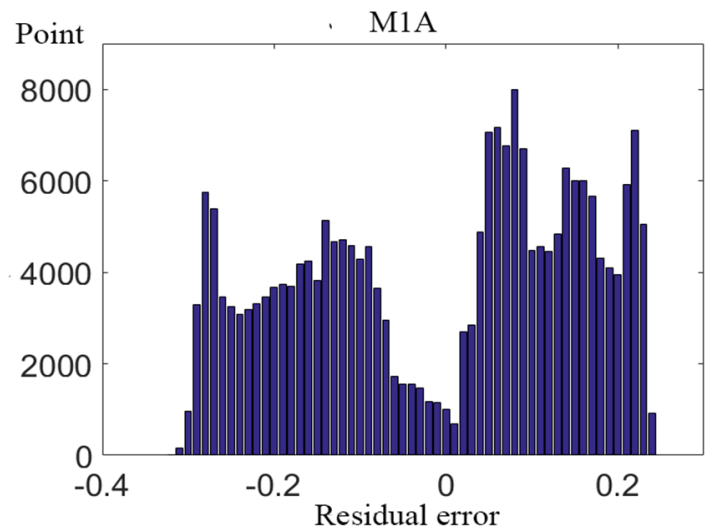

Fig.3. M1A before calibration

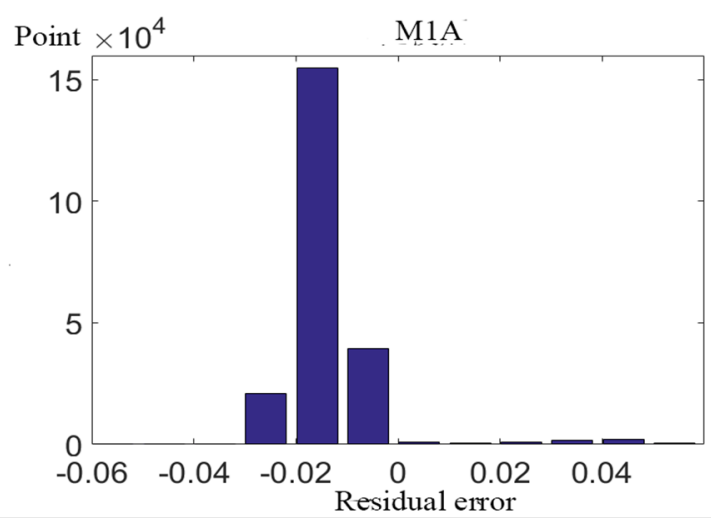

Fig.4. M1A after calibration

As can be seen from Fig.3 and Fig.4, the residual distribution before calibration is relatively discrete, and residual error of M1A after calibration is mainly distributed around -0.02 .

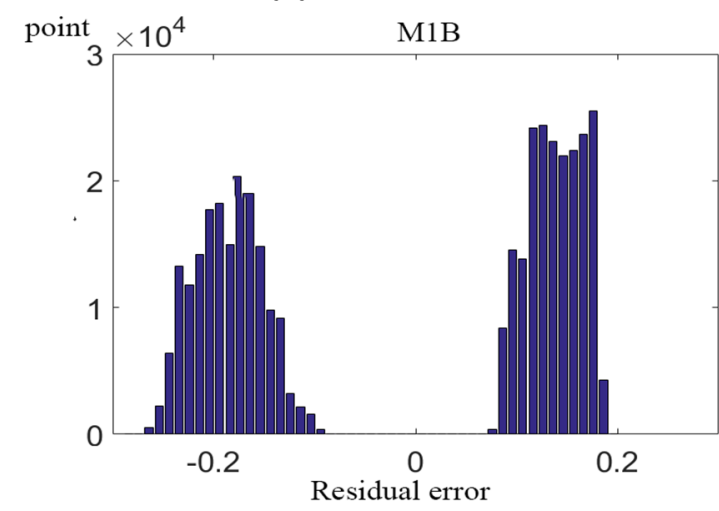

Fig.5. M1B before calibration

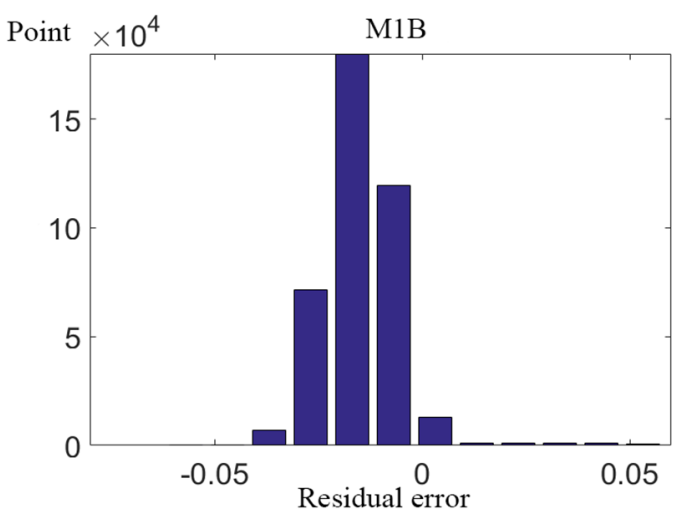

Fig.6. M1B after calibration
As can be seen from Fig.5 and Fig.6, the residual distribution before calibration is mainly in -0.2 or 0.16 , and that of M1A after calibration is mainly around -0.02 .

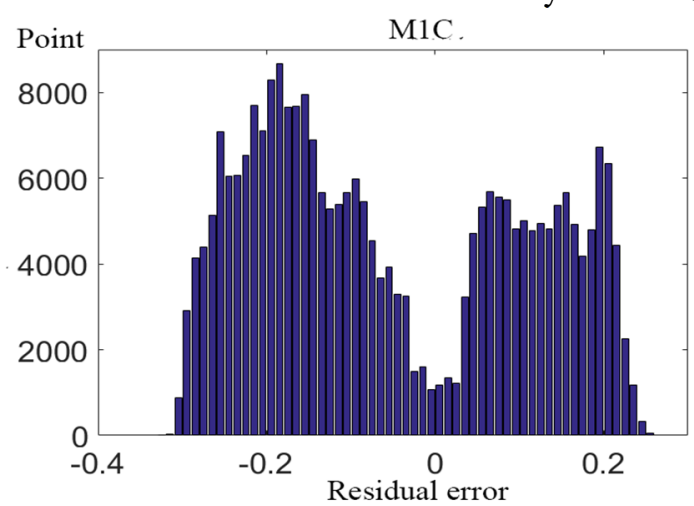

Fig.7. M1C before calibration

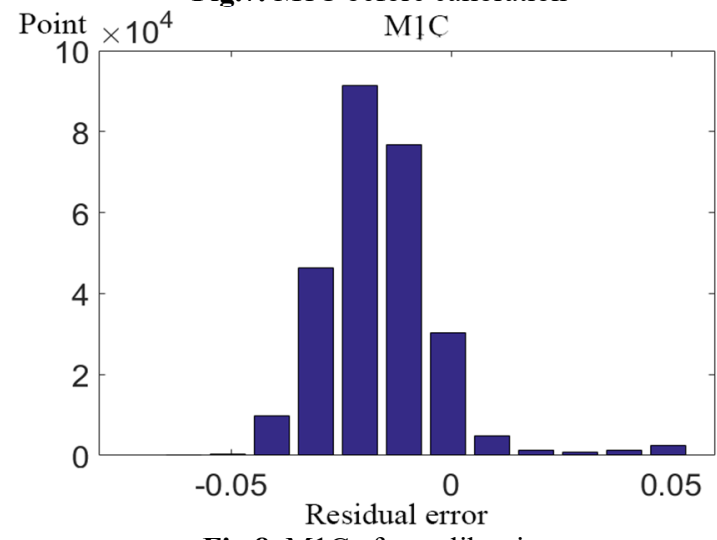

Fig.8. M1C after calibration

As can be seen from Fig.7 and Fig.8, the residual distribution before calibration is between -0.27 and 0.2 , after calibration, the residual error of $\mathrm{M} 1 \mathrm{C}$ is mainly distributed around -0.025 .

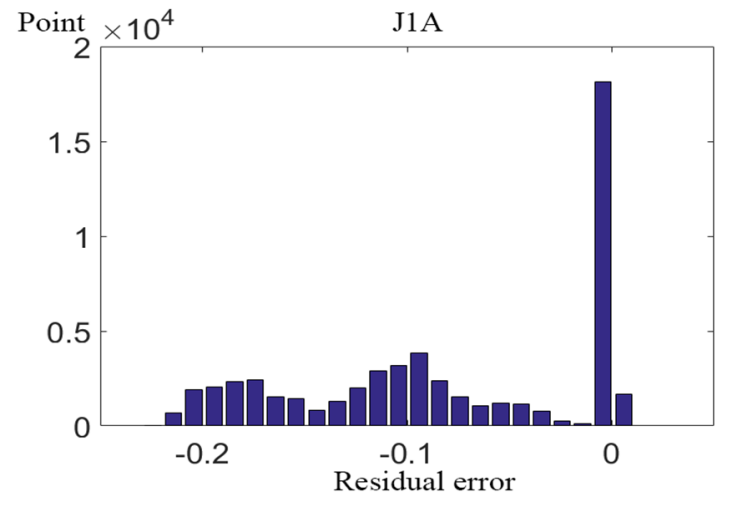

Fig.9. J1A before calibration

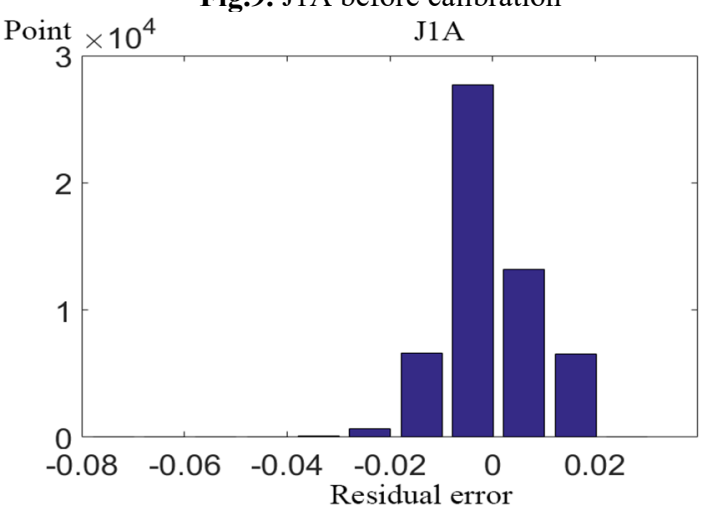

Fig.10. J1A after calibration 
As can be seen from Fig.9 and Fig.10,the residual distribution before calibration is discrete, and the residual after calibration is mainly distributed around -0.01 .

Through the inspection of the same feature plane scanned by the point cloud visualization software in different directions, it can be found that the point clouds in the same place repeatedly scanned before calibration, the point clouds of the same point after compensation for boresight error coincide well, and the calibration effect is better.

\section{Conclusion}

This paper describes a calibration method of the MMS with known feature plane, and organizes the calibration experiment. Using the known feature plane parameters and the laser point cloud positioning equation, this method establishes the Gasuu-Helmert model of the boresight parameters, and deduces the solution process of the model with the classical least square principle, and calculates the boresight error of the MMS. The residual error before and after the solution shows that the method has the characteristics of high calibration accuracy, strong reliability and simple layout. The feature plane is less affected by occlusion, speed and other factors, so it is easy to identify and extract, and has certain advantages compared with other features. It is proved that this method has extreme practicability and is an excellent method for checking and calibrating parameters.

\section{Reference}

1. J Yu, S Liu. 3S Integration technology Based LD2000 Series Mobile Mapping System and Its Applications. Bulletin of Surveying and Mapping, (7),119-120,(2013).

2. D Li. Mobile Mapping technology and its application. Geospatial Information.,4(4),1-5, (2006).

3. B Yang, W Zheng, Q Li. Jonathan Li. Automated extraction of street-scene objects from mobile lidar point clouds. International Journal of Remote Sensing, 33(18), 5839-5861, (2012).

4. R Wan, Y Huang, R Xie. Combined lane mapping using a mobile mapping system. Remote Sensing, (11), 305-330, (2019).

5. L Ma, Y Li, J Li. Mobile laser scanned point-clouds for road object detection and extraction: a review. Remote Sensing,(10),1531-1564, (2018)

6. T Chan, C Glennie. Multi-feature based boresight se ;f-calibration of a terrestrial mobile mapping system. ISPRS Journal of Photogrammetry and Remote Sensing, 82, 112-124, (2013).

7. C Glennie. A Kinematic Terrestrial LIDAR Scanning System. InsideGNSS, 2(7), 22-31, (2007).

8. Z Wang, A Zhang, S Wang. Airborne Radar Calibtation System based on the overlap strip. Chinese Journal of Lasers, 41(2), 1-8, (2014).

9. L Yao, Z Wang, H Sun. Design and implementation of Vehicle Laser Scanner is external parameter calibration. Journal of Tongji University,41(11), 161166, (2015).

10. X Meng, S Hu, A Zhang. A Self-Calibration method for bore-sight error of ground-based Mobile Mapping System. Chinese Journal of Lasers,41(11), 161-166, (2015). 